\title{
Desenvolvimento do Surubim do Iguaçu (Steindachneridion sp., Garavello (1991)) (Siluroidei:Pimelodiae) em ambiente escuro durante a fase inicial, alimentado com diferentes dietas
}

\section{Development of the Iguaçu's Surubim (Steindachneridion sp., Garavello (1991)) (Siluroidei:Pimelodiae) in darkness during the initial phase, fed with different diets}

\author{
Aldi Feiden ${ }^{1 *}$; Carmino Hayashi²; Wilson R. Boscolo ${ }^{3}$; Altevir Signor ${ }^{4}$
}

Resumo

\begin{abstract}
O experimento verificou o desenvolvimento de larvas de surubim do Iguaçu (Steindachneridion sp), submetidas a diferentes dietas em ambiente não iluminado. Foram utilizados aquários de 35 litros, em um delineamento inteiramente casualizado, com cinco tratamentos e quatro repetições. Colocaram-se 15

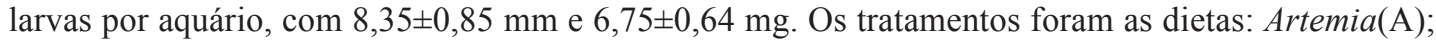
ração(R); zooplâncton(Z); Artemia+ração(A+R); e zooplâncton+ração (Z+R). Após 29 dias, os resultados mostraram que os tratamentos $\mathrm{A}+\mathrm{R}$ e $\mathrm{Z}+\mathrm{R}$ obtiveram pesos médios finais de 1036 e $859 \mathrm{mg}$, significativamente maiores $(\mathrm{P}<0,01)$, que $\mathrm{A}$ e $\mathrm{Z}$, com 536 e $270 \mathrm{mg}$, respectivamente. No tratamento $\mathrm{R}$ não houve sobrevivência de larvas, e apresentou alta taxa de canibalismo (71\%), o qual foi também observado nos outros tratamentos, em menores taxas. Pode-se concluir que a associação de alimentos vivos e dietas artificiais em ambientes escuros apresenta bom desenvolvimento mas não reduz o canibalismo. Palavras-chave: Larvicultura. Alimentação inicial. Condições ambientais. Ritmo alimentar.
\end{abstract}

\begin{abstract}
The experiment verified the development of Iguaçu's surubim (Steindachneridion sp.) larvae, submitted to different diets in darkness. 35L-aquariums were used, in a completely randomized design, with five treatments and four repetitions. Each aquarium had 15 larvae with $8,35 \pm 0,85 \mathrm{~mm}$ and 6,75 $\pm 0,64 \mathrm{mg}$, and the treatments were the following diets: $\operatorname{Artemia}(\mathrm{A})$; ration(R); zooplankton(Z); $\operatorname{Artemia}+$ ration(A+R); and zooplankton+ration $(\mathrm{Z}+\mathrm{R})$; After 29 days, the treatments $\mathrm{A}+\mathrm{R}$ and $\mathrm{Z}+\mathrm{R}$ was $1036 \mathrm{mg}$ and $859 \mathrm{mg}$, respectively, significantly higher $(\mathrm{P}<0,01)$, that $\mathrm{A}$ and $\mathrm{Z}$, with 536 and $270 \mathrm{mg}$, respectively. In the treatment $\mathrm{R}$ there was no larvae survival, and presented high cannibalism (71\%), which was also observed in other treatments, in smaller rates. This results led to the conclusions that the association of live and artificial diets in darkness provided best development, but it does not reduce the cannibalism.

Key words: Larviculture. Initial feeding. Environmental conditions. Alimentary rhythm.
\end{abstract}

\footnotetext{
1 Engenheiro Agrônomo, Professor Adjunto - Universidade Estadual do Oeste do Paraná -Campus de Toledo - Unioeste - Rua da Faculdade,645 CEP 85903000, Toledo/PR. E-mail:aldi@unioeste.br.

2 Biólogo - Prof. Titular, Programa de Pós-Graduação em Ecologia de Ambientes Aquáticos Continentais - Universidade Estadual de Maringá. PEA-UEM. E-mail: chayashi@netsite.com.br

3 Zootecnista, Professor Adjunto - Universidade Estadual do Oeste do Paraná -Campus de Toledo - Unioeste - Rua da Faculdade,645 CEP 85903000, Toledo/PR. E-mail:wrboscolo@unioeste.br

4 Acadêmico de Engenharia de Pesca - Universidade Estadual do Oeste do Paraná -Campus de Toledo - Unioeste.

* Autor para correspondência
} 


\section{Introdução}

Como a fase inicial de desenvolvimento das espécies reofílicas é hoje o principal ponto de estrangulamento para a produção de juvenis de importância para a pesca e cultivo (Basile-Martins, 1984), o uso de diferentes alimentos e condições ambientais permite obter conhecimentos sobre a biologia das espécies.

Dentre a ictiofauna neotropical destacam-se os pimelodídeos, geralmente espécies de grande porte e muito afetados no seu ambiente natural pelas atividades antrópicas (AGOSTINHO; ZALEWSKI, 1996). Para as suas espécies, poucas informações existem sobre a alimentação e desenvolvimento inicial, destacando-se os trabalhos realizados em laboratório por Behr (1997) e Furuya (2001) com pintado (Pseudoplatystoma corruscans), Kossowski (1991) com cachara (Pseudoplatystoma fasciatum), Zaniboni Filho e Barbosa (1992) com jaú (Paulicea luetkeni), Luz et al. (2001) e Luz e Zaniboni Filho (2002) com mandi-amarelo (Pimelodus maculatus), e em ambiente natural o trabalho de Rossi (2001) com jurupecê (Sorubim lima).

Os fatores ambientais são determinantes para o desenvolvimento de larvas de peixes, e influenciam sobremaneira a alimentação nos estágios iniciais do desenvolvimento.

A maioria das espécies de peixes é orientada visualmente (BARAS; JOBLING, 2002), e a redução de luminosidade, mudança de contraste da luz, fotoperíodo e turbidez da água afetam a seleção dos alimentos e a predação intra-específica, influenciando o crescimento e a sobrevivência das larvas na fase inicial de desenvolvimento. Nass e Iglesias (1996) afirmam que a distribuição das larvas no ambiente ocorre pela procura de áreas de luminosidade ótimas, que permitam o melhor conforto.

A utilização de tanques com alteração do espectro luminoso na fase inicial foi pesquisado por Ostrowski (1989), Britz e Pienaar (1992), Appelbaum e McGeer (1998), Baras et al. (1998) e Pedreira e SipaúbaTavares (2001).
As larvas de peixes geralmente se alimentam inicialmente de zooplâncton (GERKING, 1994), o qual fornece os nutrientes essenciais para o desenvolvimento, como alto teor de aminoácidos, enzimas e água (HERBERT, 1978).

Vários autores citam o fornecimento de zooplâncton, mas Radünz Neto (1999) ressalva que o monocultivo de espécies zooplanctônicas torna a larvicultura onerosa e o plâncton silvestre coletado por redes apresenta grande variabilidade na sua composição e densidade ao longo do tempo. $\mathrm{O}$ uso de Artemia tem sido preconizado com bons resultados por Verischelle et al. (1990), Fermin e Bolívar (1991) e Stickney (1994).

A combinação de dietas naturais e artificiais é muito utilizada, sendo que entre os principais trabalhos destacam-se Verreth e Van Tongeren (1989), Fermin e Bolívar (1991), Kerdchuen e Legendre (1995), Rosenlund, Stoss e Talbot (1997). A associação de dietas proporciona redução de canibalismo (PIENAAR, 1990) e permite um desenvolvimento mais homogêneo e maior sobrevivência.

O surubim do Iguaçu (Steindachneridion sp.) é o maior pimelodídeo encontrado no rio Iguaçu, endêmico da bacia e restrito ao baixo Iguaçu (SEVERI; CORDEIRO, 1994), e cuja captura está abaixo de $0,001 \%$, sendo considerada rara e com riscos de extinção (AGOSTINHO et al., 2002a). Agostinho et al. (2002b) recomendam ações no ambiente natural para as espécies vulneráveis como o levantamento das principais áreas de desova e sua proteção e, inclusive, segundo Agostinho et al. (2002a), realizar peixamentos, desde que para isto tenha-se um absoluto controle genético do plantel de reprodutores, evitando-se a redução da variabilidade genética, e portanto, faz-se necessário o conhecimento de sua ecologia.

A espécie apresenta bom potencial para cultivo, apresentando bom desenvolvimento e alto rendimento de carcaça (FEIDEN et al., 2001a, 2001b), mas existem poucas informações sobre sua biologia 
(NAKATANI et al., 2001), alimentação e influência dos fatores ambientais sobre seu desenvolvimento, que permitam a produção de juvenis.

Este trabalho teve por objetivo avaliar o desenvolvimento de larvas de Steindachneridion sp., submetidas a diferentes dietas e suas combinações em aquários mantidos em ambiente escuro.

\section{Material e Métodos}

O experimento foi realizado nas instalações do Laboratório de Aqüicultura da Universidade Estadual do Oeste do Paraná, campus de Toledo, no período de 08 de outubro a 06 de novembro de 2002. As larvas utilizadas, com as características: 48 horas após a eclosão, comprimento e peso inicial de $8,35 \pm 0,85 \mathrm{~mm}$ e $6,75 \pm 0,64 \mathrm{mg}$, respectivamente, foram provenientes de desova induzida realizada pela Estação Experimental de Estudos Ictiológicos de Segredo, da Companhia Paranaense de Energia (Copel).

O delineamento experimental foi o inteiramente casualizado com cinco tratamentos e quatro repetições, colocando-se 15 larvas por unidade experimental, em 20 aquários com capacidade para 35 litros de volume útil, os quais receberam aeração constante através de um sistema ligado a um soprador central. A bateria de aquários foi coberta por lona de polietileno preta, durante todo o período experimental, sendo retirada a lona parcialmente para a sifonagem dos aquários e alimentação das larvas. Os tratamentos foram Artemia sp (A); ração (R); zooplâncton (Z); Artemia sp e ração $(A+R)$ e zooplâncton e ração $(Z+R)$.

Para a eclosão dos cistos de Artemia sp foram utilizadas incubadoras com capacidade de 2 litros de água, dotadas de aeração constante, abastecidas com água com salinidade de $30 \%$, e mantidas sob iluminação e temperatura constante. Após a eclosão realizou-se a filtragem em rede de $48 \mathrm{~mm}$ e o fornecimento às larvas. $\mathrm{O}$ zooplâncton foi coletado diariamente nos viveiros do Centro de Pesquisas em Aqüicultura Ambiental (CPAA/IAP), de Toledo através de rede de arrasto com abertura de $70 \mathrm{~mm}$.
A ração foi formulada com $38 \%$ de proteína bruta (PB) (Tabela 1), e os ingredientes moídos de forma a apresentarem granulometria de $0,5 \mathrm{~mm}$ de diâmetro.

Tabela 1. Composição percentual das rações fornecidas às larvas de surubim do Iguaçu (Steindachneridion sp.) durante a fase inicial de desenvolvimento.

\begin{tabular}{lc}
\hline \multicolumn{1}{c}{ INGREDIENTES } & $\%$ \\
\hline Antioxidante (BHT) & 0,02 \\
Calcário calcítico & 0,60 \\
Fosfato bicálcico & 1,46 \\
Farelo de soja & 27,66 \\
Farinha de peixe & 30,23 \\
Farinha de visceras & 12,50 \\
Milho & 17,51 \\
Óleo de soja & 8,52 \\
Suplemento (min+vit) ${ }^{1}$ & 1,00 \\
Sal comum & 0,50 \\
\hline TOTAL NuTRIENTES & 100 \\
\hline & $(\%)$ \\
\hline Energia digestível $(\mathrm{Kcal} / \mathrm{kg})^{2}$ & 3600 \\
Proteina bruta & 38,00 \\
Gordura & 17,56 \\
Fibra bruta & 1,69 \\
Fósforo total & 1,56 \\
Cálcio total & 3,00 \\
Linoleico & 5,59 \\
Lisina & 2,42 \\
Metonina+cistina & 1,55 \\
TOTAL & $\mathbf{1 0 0}$ \\
\hline
\end{tabular}

${ }^{1}$ Níveis de garantia por quilograma do produto (Rovimix peixes): Vit. A, 500.000UI; Vit. D, 200.000UI; Vit. E, 5.000mg; Vit. K3, 1.000mg; Vit. B1, 1.500mg; Vit. B2, 1.500mg; Vit. B6, $1.500 \mathrm{mg}$; Vit. B12, 4.000mg; Ác. Fólico, 500mg; Pantotenato Ca, 4.000mg; Vit. C, 15.000mg; Biotina, 50mg; Inositol, 10.000; Nicotinamida, 7.000; Colina, 40.000mg; Co, 10mg; Cu, 500mg; Fe, 5.000mg; I, 50mg; Mn, 1500mg; Se, 10mg; Zn, 5.000mg. ${ }^{2}$ Baseados nos valores de energia e digestível proposto por (NRC1993).

Os alimentos foram fornecidos três vezes ao dia (8:00; 13:30 e 18:00 horas), precedido de sifonagem da água do fundo dos aquários para retirada de sobras de alimentos e fezes, na proporção de $10 \%$ do volume na primeira semana, e de $30 \%$ no restante do período experimental. A densidade média de organismos fornecidos diariamente foi de 42.000 e 120.000 indivíduos para zooplâncton e Artemia sp, respectivamente, e a ração foi fornecida ad libitum.

Os parâmetros físico-químicos da água como oxigênio dissolvido (mg. $\left.\mathrm{L}^{-1}\right), \mathrm{pH}$ e condutividade 
elétrica $\left(\mathrm{mS}^{\mathrm{cm}}{ }^{-1}\right)$ foram monitorados uma vez por semana no período da tarde, enquanto que a temperatura $\left({ }^{\circ} \mathrm{C}\right)$ foi medida duas vezes ao dia (manhã e tarde) durante o experimento. Os parâmetros bióticos, como mortalidade, canibalismo e sobrevivência foram monitorados durante o período experimental e ao final do experimento todos os indivíduos foram medidos e pesados para avaliação dos parâmetros bióticos. O modelo estatístico utilizado para análise dos dados foi o seguinte:

$\mathrm{Y}_{\mathrm{ij}}=\mathrm{m}+\mathrm{Ti}+\mathrm{eij}$

em que:

$\mathrm{Y}_{\mathrm{ij}}=$ observação referente ao aquário $\mathrm{j}$ onde utilizouse o tratamento i;

$\mathrm{m}=$ constante geral;

$\mathrm{Ti}=$ efeito do tratamento $\mathrm{i}$, onde $\mathrm{i}: \mathrm{A} ; \mathrm{R} ; \mathrm{Z} ; \mathrm{A}+\mathrm{R} ; \mathrm{Z}+\mathrm{R}$;
Eij = erro aleatório associado a observação $Y_{i j}$

Os dados foram submetidos à análise de variância e ao teste de Tukey para diferenças estatísticas a 1,00\% de probabilidade, pelo programa computacional Minitab for Windows ${ }^{\circledR}$ versão 10.1. Os valores de mortalidade, canibalismo e sobrevivência foram transformados pela expressão $y=\operatorname{arcsen} " x / 100$, sendo $x$ o valor destas características expresso em percentagem.

\section{Resultados e discussão}

Os parâmetros físico-químicos monitorados ao longo do experimento, mostrados na Tabela 2, não mostraram variações que possam ter influenciado nos resultados, quanto ao desempenho e sobrevivência, pois todos os fatores permaneceram dentro da faixa de conforto para as espécies tropicais, segundo Sipaúba-Tavares, Bachion e Rocha (1994).

Tabela 2. Valores médios dos parâmetros físico-químicos obtidos durante o experimento com larvas de Steindachneridion sp. em ambiente sem luminosidade durante a fase inicial, alimentados com diferentes dietas.

\begin{tabular}{lccccc}
\hline Parâmetros & \multicolumn{5}{c}{ Tratamentos } \\
\cline { 2 - 6 } & $\mathrm{A}$ & $\mathrm{R}$ & $\mathrm{Z}$ & $\mathrm{A}+\mathrm{R}$ & $\mathrm{Z}+\mathrm{R}$ \\
\hline Temperatura $\left({ }^{\circ} \mathrm{C}\right)$ & $24,2 \pm 0,5$ & $24,4 \pm 0,5$ & $24,2 \pm 0,6$ & $24,2 \pm 0,5$ & $24,3 \pm 0,6$ \\
\hline Oxigênio dissolvido $\left(\mathrm{mg} \cdot \mathrm{L}^{-1}\right)$ & $7,2 \pm 0,6$ & $6,9 \pm 0,2$ & $6,7 \pm 0,4$ & $6,1 \pm 0,9$ & $5,3 \pm 1,7$ \\
Ph & $7,18 \pm 0,2$ & $7,41 \pm 0,2$ & $7,40 \pm 0,2$ & $7,08 \pm 0,2$ & $6,98 \pm 0,2$ \\
Condutividade elétrica $\left(\mu \mathrm{S} . \mathrm{cm}^{-1}\right)$ & $137 \pm 16,4$ & $118 \pm 0,7$ & $119 \pm 3,8$ & $134 \pm 4,5$ & $120 \pm 2,0$ \\
\hline
\end{tabular}

Tratamentos: A- Artemia sp.; R- Ração; Z- Zooplâncton; A+R-Artemia e ração; Z+R- Zooplâncton e ração.

Tabela 3. Valores médios dos parâmetros bióticos ao final do experimento com larvas de Steindachneridion sp.

\begin{tabular}{lcccccc}
\hline Parâmetros & \multicolumn{5}{c}{ Tratamentos } & \multirow{2}{*}{ CV } \\
\cline { 2 - 5 } & $\mathrm{A}$ & $\mathrm{R}$ & $\mathrm{Z}$ & $\mathrm{A}+\mathrm{R}$ & $\mathrm{Z}+\mathrm{R}$ & \\
\hline Comprimento inicial (mm) & $8,35 \pm 0,85$ & $8,35 \pm 0,85$ & $8,35 \pm 0,85$ & $8,35 \pm 0,85$ & $8,35 \pm 0,85$ & 0,85 \\
Comprimento final (mm) & $36,7 \pm 0,4^{\mathbf{a}}$ & $*$ & $27,4 \pm 2,8^{\mathbf{b}}$ & $43,5 \pm 1,4^{\mathbf{c}}$ & $40,1 \pm 1,8^{\mathbf{a c}}$ & 1,82 \\
Peso inicial (mg) & $6,75 \pm 0,64$ & $6,75 \pm 0,64$ & $6,75 \pm 0,64$ & $6,75 \pm 0,64$ & $6,75 \pm 0,64$ & 0,64 \\
Peso final (mg) & $536 \pm 26,5^{\mathbf{a}}$ & $*$ & $270 \pm 98,1^{\mathbf{b}}$ & $1036 \pm 54,7^{\mathbf{c}}$ & $859 \pm 104^{\mathbf{c}}$ & 77,70 \\
Ganho de peso diário (mg/dia) & $18,3 \pm 0,9^{\mathbf{a}}$ & $*$ & $8,4 \pm 2,5^{\mathbf{b}}$ & $35,5 \pm 1,9^{\mathbf{a}}$ & $29,4 \pm 3,6^{\mathbf{c}}$ & 2,42 \\
Sobrevivência (\%)** & $85 \pm 8,4^{\mathbf{a}}$ & $00,0^{\mathbf{b}}$ & $70,11 \pm 7,6^{\mathbf{a}}$ & $76,7 \pm 11,6^{\mathbf{a}}$ & $63,3 \pm 8,6^{\mathbf{a}}$ & 12,14 \\
Mortalidade (\%) & $1,68 \pm 3,4^{\mathbf{a}}$ & $28,3 \pm 14,8^{\mathbf{b}}$ & $6,7 \pm 7,7^{\mathbf{a b}}$ & $1,7 \pm 3,4^{\mathbf{a}}$ & $1,7 \pm 3,4^{\mathbf{a}}$ & 7,89 \\
Canibalismo (\%) & $13,4 \pm 9,4^{\mathbf{a}}$ & $71,7 \pm 14,8^{\mathbf{b}}$ & $23,3 \pm 20,7^{\mathbf{a}}$ & $21,6 \pm 8,4^{\mathbf{a}}$ & $35,1 \pm 9,9^{\mathbf{a b}}$ & 13,47 \\
\hline
\end{tabular}

Tratamentos: A- Artemia sp.; R- Ração; Z- Zooplâncton; A+R-Artemia e ração; Z+R-Zooplâncton e ração. Valores na mesma linha seguidos de mesma letra não diferem estatisticamente $(\mathrm{P}>0,01)$ pelo teste de Tukey

* Não houveram sobreviventes no tratamento.

** A densidade inicial foi de 15 larvas por unidade experimental. 
Ao final do experimento os tratamentos apresentaram diferenças significativas $(\mathrm{P}<0,01)$ entre si, como demonstrado na Tabela 3.

O tratamento $\mathrm{A}+\mathrm{R}$ proporcionou o melhor desempenho das larvas, demonstrando a importância da Artemia no início da alimentação exógena, devido ao seu alto valor biológico e pequeno tamanho, o que permita que seja o primeiro alimento ingerido pelas larvas, como constatado por Kossowski (1991). Naess, Germainhenry e Naas (1995) mostraram que o uso de Artemia promove maior crescimento e sobrevivência, quando comparado a alimentação com zooplâncton, e Fermin e Bolívar (1991), Kerdchuen e Legendre (1995) e Soares, Hayashi e Gonçalves (2000) demonstraram que o uso de Artemia associado com ração promoveu taxas de crescimento e sobrevivência maiores que plâncton associado com ração, para larvas de Clarias macrocephalus, Clarias gariepinus e Carassius auratus, respectivamente. Qin et al. (1997) recomendam inicialmente o fornecimento de Artemia e numa segunda fase sua substituição por alimento seco.

O menor desempenho final das larvas do tratamento $\mathrm{Z}$ demonstra que apesar de inicialmente o desenvolvimento ser satisfatório, pelo fato do zooplâncton ser um alimento de alto valor biológico e liberar enzimas proteolíticas (DABROWSKI; GLOGOWSKI, 1977; HERBERT, 1978), com o desenvolvimento das larvas, estas passaram a ter preferência pelos alimentos inertes (UFODIKE; WADA, 1991).

Os resultados referentes a sobrevivência mortalidade e canibalismo, (Tabela 3), mostram as maiores taxas de canibalismo $(71,7 \%)$ e mortalidade $(28,3 \%)$ no tratamento $\mathrm{R}$, que resultou na eliminação de todos os indivíduos alimentados somente com dieta artificial, demonstra a importância dos organismosalimento na fase inicial do desenvolvimento das larvas. Walford e Lam (1993) também constataram o baixo desempenho das dietas inertes e atribuíram isto a composição e características dos alimentos, e a incapacidade de digestão pelas larvas, e Segner et al. (1993) verificaram inanição em larvas de Clarias alimentadas somente com ração.

As taxas de sobrevivência encontradas, exceto no tratamento $\mathrm{R}$, demonstram que o ambiente sem luminosidade pode ter reduzido a agressividade, permitindo um melhor desenvolvimento. Britz e Pienaar (1992) verificaram que para Clarias gariepinus cultivados sob luz intensa, os indivíduos se movimentam para o fundo dos tanques e a competição e a agressividade aumentam. Baras et al. (1999) relatam que a criação de pimelodídeos em escuridão permanente resulta em crescimento mais rápido e menos heterogêneo, especialmente quando a alimentação é fornecida continuamente.

O canibalismo ocorreu em todos os tratamentos, mas diferindo estatisticamente para o tratamento $\mathrm{R}$, e estes resultados mostram que a predação intraespecífica é um fenômeno freqüente nas fases iniciais do desenvolvimento dos peixes, o que é relatado por Baras e Jobling (2002), e ocorre também em ambientes não iluminados, pois de acordo com Baras et al. (1999) e Lamb (2001), como os pimelodídeos se orientam pelo tato e por quimiosensores, localizados nos barbilhões, não necessitam de luz para a predação.

A alta taxa de canibalismo do tratamento $\mathrm{R}$, provavelmente ocorreu pela vulnerabilidade das larvas famintas, as quais tornam-se muito suscetíveis devido a inanição e não conseguem escapar dos predadores, o que é relatado por Smith e Ready (1991).

$\mathrm{O}$ alto peso final encontrado nos tratamentos $\mathrm{A}+\mathrm{R}$ e $\mathrm{Z}+\mathrm{R}$, quando comparados ao cultivo da espécie em ambiente iluminado, nas mesmas condições, realizado por Feiden (2003), que obteve $659 \mathrm{mg}$ e $571 \mathrm{mg}$ para larvas alimentadas com Artemia mais ração e zooplâncton mais ração, respectivamente, confirma a importância da ausência de luz sobre o desenvolvimento do surubim do Iguaçu Steindachneridion sp. 


\section{Conclusão}

Podemos concluir que a espécie Steindachneridion sp., alimentada com a combinação da dieta natural e artificial, em ambiente escuro, apresenta um desenvolvimento homogêneo e mais rápido permitindo a redução do canibalismo e maior sobrevivência para as larvas.

\section{Referências}

AGOSTINHO, A. A.; PAVANELLI, C. S.; SUZUKI, H. I. ; LATINI, J. D.; GOMES, L. C. HAHN, N. S.; FUGI, R.; DOMINGUES, W. M. Reservatório de Salto Caxias: bases ecológicas para o manejo. Maringá: UEM/Nupelia/Copel, 2002a. $272 p$

AgOstinho, A. A. et al. Reservatório de Salto Caxias: questionamentos feitos pelo PROCAXIAS em relação ao contido no Plano Diretor de Caxias e outros posicionamentos. Parecer Técnico. Maringá: UEM/ Nupelia, 2002b. 25p. Mimeo.

AGOSTINHO, A. A.; ZALESWSKI, A planície alagável do alto rio Paraná: importância e preservação. Maringá: Eduem, 1996. 100p.

APPELBAUM, S.; McGEER, J. C. Effect of a diet and light regime on growth and survival of African catfish (Clarias gariepinus) larvae and early juveniles. Aquaculture Nutrition, Oxford, v.4, p.157-164. 1998.

BARAS, E.; TISSIER, F.; WESTERLOPPE, L.; MELARD, C; PHILIPPART, J. C. Feeding in darkness alleviates density-dependent growth of juvenile vundu catfish Heterobranchus longifilis (Clariidae). Aquatic Living Resources, Montrouge, v.11, p.335-340, 1998.

BARAS, E.; TISSIER, F.; PHILIPPART, J. C.; MEALARD, C. Sibling cannibalism among juvenile vundu under controlled conditions. II. Effect of body weight and environmental variables on the periodicity and intensity of type II cannibalism. Journal of Fish Biology, London, v.54, p. 54-118, 1999.

BARAS, E.; JOBLING, M. Dynamics of intracohort cannibalism in cultured fish. Aquaculture Research. England, v.33, p.461-479, 2002.

BASILE-MARTINS, M. A. Criação de organismos para alimentação de larvas de peixes. In: SIMPÓSIO BRASILEIRO DE AQUICULTURA, 1., 1984, São Carlos. Anais... São Carlos: Abraq, 1984. p.97-100.
BEHR, E. R. Efeitos de diferentes dietas sobre a sobrevivência e crescimento de larvas de Pseudoplatystoma corruscans (Agassiz, 1829)(Pisces:Pimelodidae). 1997. Dissertação (Mestrado) - Universidade Estadual de Maringá, Maringá.

BRITZ, P. J.; PIENAAR, A. G. Laboratory experiments on the effect of light and cover on the bahaviour and growth of African catfish Clarias gariepinus (Pisces: Clariidae). Journal of Zoology, England, v.227, p.44-62, 1992.

DABROWSKI, K.; GLOGOWSKI, J. Studies on the role of exogenous proteolytic enzymes in digestion processes in fish. Hydrobiologia, The Hague-Holanda, v.54, p.129-134, 1977.

FEIDEN, A. Desenvolvimento de larvas de surubim do Iguaçu Steindachneridion sp. Steindachneridion sp. (Eigenmann \& Eigenman, 1919) (Siluroidei: Pimelodidae), submetidas a diferentes dietas e condições ambientais. 2003. Tese (Doutorado) - Universidade Estadual de Maringá, Maringá.

FEIDEN, A.; et al. Proporções corporais do surubim do Iguaçu Steindachneridion sp (Pisces:Pimelodidae) em três diferentes idades. In: CONGRESSO BRASILEIRO DE ENGENHARIA DE PESCA, 12., 2001, Foz do Iguaçu. Anais... Foz do Iguaçu: AEP/Sul, 2001a. CD-Rom.

FEIDEN, A. et al., Desenvolvimento do surubim Steindachneridion $\mathrm{sp}$ em tanques-rede, noreservatório da Hidrelétrica de Salto Caxias. In: CONGRESSO BRASILEIRO DE ECOLOGIA, 5., 2001, Porto Alegre. Resumos... Porto Alegre: SBE, 2001b. p.22.

FERMIN, A. C.; BOLIVAR, M. E. C. Larval rearing of the philippine freshwater catfish, Clarias macrocephalus (Gunther) fed live zooplankton and artificial diet: apreliminary study. Bamidgeh, Nir-David, Israel, v.43, n.3, p.87094, 1991.

FURUYA, V. R. B. Alimentação e desenvolvimento do pintado Pseudoplatystoma corruscans (Agassis, 1829) (Pisces:Pimelodidae) : Macrófitas aquáticas e utilização do isótopo estável de carbono $\left({ }^{13} \mathrm{C}\right)$. (Tese:Doutorado) Universidade Estadual de Maringá. 2001.

GERKING, S. D. Larval feeding. In: GERKING, S. D. (Ed.). Feeding ecology of fish. London: Academic, 1994. p. 139170.

HERBERT, P. D. N. The populations biology of Daphnia (Crustacea: Daphnidae). Biochimica et Biophysica Acta: Review on Bioenergetics, Amsterdam, v.53, p.387-426, 1978.

KERDCHUEN, N.; LEGENDRE, M. Larval rearing of an African catfish Heterobranchus longifilis (Teleostei:Clariidae): acomparison between natural and artificial diet. Aquatic Living Resources, Montrouge, v.17, p.247-253, 1994. 
KOSSOWSKI, C. Experiências iniciales sobre la hibridización de Leiarius marmoratus (GILL, 1871) por Pseudoplatystoma fasciatum (Linnaeus) 1766 (Pisces, Siluriformes, Pimelodidae). Acta Cientifica Venezolana, v.42, p.48-50. 1991.

LAMB, C. F. Gustation and feeding behaviour. In: HOULIHAN, D. et al. (Ed.). Food intake in fish. Oxford: Blackwell Science, 2001. p. 49-87.

LUZ, R. K.; REYNALTE-TATAJE, D. A.; FERREIRA, A. A.; ZANIBONI-FILHO, E. Desenvolvimento embrionário e estagios larvais do mandi-amarelo Pimelodus maculatus. Boletim do Instituto de Pesca, São Paulo, v.27, n.1, p.49$55,2001$.

LUZ, R.K.; ZANIBONI FILHO, E. Larvicultura do mandiamarelo Pimelodus maculatus Lacépède, 1803 (Siluriformes:Pimelodidae) em diferentes densidades de estocagem nos primeiros dias de vida. Revista Brasileira de Zootecnia, Viçosa-MG, v.31, n.2, p.560-565. 2002.

NAESS, T.; GERMAINHENRY, M.; NAAS, K. E. First feeding of Atlantic halibut (Hippoglosus hippoglossus) using different combinations of Artemia and zooplankton. Aquaculture, Amsterdam, v.130, p. 235-250. 1995.

NAKATANI, K.; AGOSTINHO, A. A.; BAUMGARTNER, G.; BIALETZKI, A.; SANCHES, P. V.; MAKRAKIS, M. C.; PAVANELLI, C. S. Ovos e larvas de peixes de água doce: desenvolvimento e manual de identificação. Maringá: Eduem, 2001.378 p.

NASS, K.; IGLESIAS, J. Illumination in first feeding tanks for marine fish larvae. Aquaculture, Amsterdam, v.15, n.4, p.291-300, 1996.

OSTROWSKI, A. C. Effect of rearing tank background color on early survival of dolphin larvae. Progressive FishCulturist, Bethesda, v.51, n.3, p.161-163, 1989.

PEDREIRA, M. M.; SIPAÚBA-TAVARES, L. H. Effect of light green and dark Brown colored tanks on survival rates and development of tamaqui larvae, Colossoma macropomum (Osteichthes: Serrasalmidae). Acta Scientiarum, Maringá, v.23, n.2, p.521-525, 2001.

PIENAAR, A. G. A study of coeval sibling cannibalism in larval and juvenile fishes and its control under culture conditions. 1990. (Masters Thesis) - Rhodes University, Grahamstown,, 1990, 162 p.

QIN, J.; FAST, A. W.; DeANDA, D.; WEIDENBACH, R. P. Growth and survival of larval snakehead (Channa striatus) fed different diets. Aquaculture, Amsterdam, v.148, 105113. 1997.

RADÜNZ NETO, J. Alimento natural versus ração balanceada na larvicultura de peixes. In: REUNIÃO ANUAL DA SOCIEDADE BRASILEIRA DE ZOOTECNIA, 36., 1999, Porto Alegre. Anais... Porto Alegre: SBZ, 1999. p. 119-124.
ROSENLUND, G.; STOSS, J.; TALBOT, C. Co-feeding marine fish larvae with inert and live diets. Aquaculture, Amsterdam, v.155, p.183-191, 1997.

ROSSI, L. M. Ontogenetic diet shifts in a neotropical catfish, Sorubim lima (Scheider) from the River Parana System. Fish.Managem and Ecology, Oxford, v.8, p.141152, 2001.

SEGNER, H.; ROSCH, R.; VERRETH, J.; WITT, U. Larval nutritional physiology: studies with Clarias gariepinus, Coreogonus lavaretus and Scophthalmus. Journal of The World Aquaculture Society, Baton Rouge, v.24, p.121-134. 1993.

SEVERI, W.; CORDEIRO, A. A.M. Catálogo de peixes do rio Iguaçu. Curitiba: IAP/GTZ, 1994. 118p.

SIPAÚBA-TAVARES, L. H.; BACHION, M. A.; ROCHA, O. Estudo do crescimento populacional de três espécies zooplanctônicas em laboratório e o uso de plâncton na alimentação de alevinos de Oreochromis niloticus (tilápia) e Astyanax scabripinus paranae (lambari). Revista da Unimar, Maringá, v.16, n. 3, p.189-201, 1994.

SMITH, C.; READY, P. Cannibalism in teleost fishes. Reviews in Fish and Fisheries. London, v.1 p.41-64, 1991.

SOARES, C. M.; HAYASHI, C.; GONÇALVES, G. S. Plâncton, Artemia sp, dieta artificial e suas combinações no desnvolvimento e sobrevivência do quinguio (Carassius auratus) durante a larvicultura. Acta Scientiarum, Maringá, v.22, n.2, p.383-388. 2000.

STICKNEY, R. R. Principles of aquaculture. New York: John Willey \& Sons, 1994. 502p.

UFODIKE, E. B. C.; WADA, R.K. Feeding habits of tilápia Sarotherodon niloticus (Perciformes: Ciclidae) fry in Jos, Nigéria. Revista de Biologia Tropical, San Jose, v.39, n.2,p.189-192, 1991.

VERISCHELE, D. et al. The use of Artemia. In: BARNABÉ, G. (Ed.). Aquaculture. New York: Ellis Horwood, 1990. v.1, p. 246-263.

VERRETH, J.; VAN TONGEREN, M. Weaning time in Clarias gariepinus (Burchell) larvae. Aquaculture, Amsterdam, v.83, p.81-88, 1989.

WALFORD, J.; LAM, T. J. Development of digestive tract and proteolytic enzyme activity in seabass (Lates calcifer) larvae and juveniles. Aquaculture, Amsterdam, v.109, p.187-205. 1994.

ZANIBONI-FILHO, E.; BARBOSA, N. D. C. Larvicultura na CEMIG. In: ENCONTRO ANUAL DE AQUICULTURA DE MINAS GERAIS, 10., 1992, Belo Horizonte. Anais... Belo Horizonte: Cemig, 1992. p.36-42. 